\title{
Role of Locoregional Treatment in Vulvar Cancer With Pelvic Lymph Node Metastases: Time to Reconsider FIGO Staging?
}

\author{
Ashwin Shinde, MDa; Richard Li, MDa; Arya Amini, MDa; Yi-Jen Chen, MD, PhDa; Mihaela Cristea, MD; \\ Wenge Wang, MD, PhD'; Mark Wakabyashi, MDc; Ernest Han, MD, PhDc; Catheryn Yashar, MDd; \\ Kevin Albuquerque, MDiं Sushil Beriwal, MD;; and Scott Glaser, $\mathrm{MD}^{\mathrm{a}}$
}

\begin{abstract}
Background: Vulvar cancer with pelvic nodal involvement is considered metastatic (M1) disease per AJCC staging. The role of definitive therapy and its resulting impact on survival have not been defined. Patients and Methods: Patients with pelvic lymph node-positive vulvar cancer diagnosed in 2009 through 2015 were evaluated from the National Cancer Database. Patients with known distant metastatic disease were excluded. Logistic regression was used to evaluate use of surgery and radiation therapy (RT). Overall survival (OS) was evaluated with logrank test and Cox proportional hazards modeling (multivariate analysis [MVA]). A 2-month conditional landmark analysis was performed. Results: A total of 1,304 women met the inclusion criteria. Median follow-up was 38 months for survivors. Chemotherapy, RT, and surgery were used in $54 \%, 74 \%$, and $62 \%$ of patients, respectively. Surgery was associated with prolonged OS (hazard ratio [HR], 0.58; $P<.001$ ) but had multiple significant differences in baseline characteristics compared with nonsurgical patients. In patients managed nonsurgically, RT was associated with prolonged OS (HR, 0.66; $P=.019)$ in MVA. In patients undergoing surgery, RT was associated with better OS (3-year OS, 55\% vs 48\%; $P=.033$ ). Factors predicting use of RT were identified. MVA revealed that RT was associated with prolonged OS ( $H R, 0.75 ; P=.004)$. Conclusions: In this cohort of women with vulvar cancer and positive pelvic lymph nodes, use of RT was associated with prolonged survival in those who did not undergo surgery. Surgery followed by adjuvant RT was associated with prolonged survival compared with surgery alone.
\end{abstract}

J Natl Compr Canc Netw 2019;17(8):922-930 doi: $10.6004 /$ jnccn.2019.7288

aDepartment of Radiation Oncology, ${ }^{b}$ Department of Medical Oncology, and 'Department of Gynecologic Oncology, City of Hope National Medical Center, Duarte, California; ${ }^{\mathrm{d} D e p a r t m e n t ~ o f ~ R a d i a t i o n ~ O n c o l o g y, ~ U n i v e r s i t y ~ o f ~ C a l i f o r n i a ~}$ San Diego, La Jolla, California; 'Department of Radiation Oncology, UT Southwestern Medical Center, Dallas, Texas; and fDepartment of Radiation Oncology, University of Pittsburgh Medical Center, Pittsburgh, Pennsylvania.

\section{Background}

Vulvar cancer is the fourth most commonly diagnosed gynecologic cancer in the United States, with an estimated 6,190 cases annually; it is also the fourth most lethal gynecologic cancer, with an estimated 1,200 deaths per year. ${ }^{1}$ Based on the 8th edition of the AJCC Cancer Staging Manual, patients with pelvic lymph node metastasis are classified as having metastatic (M1) disease. ${ }^{2}$ In these patients, the NCCN Clinical Practice Guidelines in Oncology (NCCN Guidelines) for Vulvar Cancer suggest consideration of radiation therapy (RT) with concurrent chemotherapy in larger T2 or T3 lesions not amenable to up-front organ-sparing surgery. ${ }^{3}$ However, minimal published data, either retrospective or prospective, exists for patients with positive pelvic lymph nodes to guide optimal management. One small retrospective series of 20 patients suggests reasonable overall survival (OS) and disease-specific survival in patients with pelvic nodal involvement. ${ }^{4}$ Given the paucity of data for this clinical situation, we used the National Cancer Database (NCDB) to evaluate the impact of definitive treatment in patients with vulvar cancer and positive pelvic lymph nodes.

\section{Patients and Methods}

\section{Data Source}

The NCDB, sponsored by the American College of Surgeons and American Cancer Society, includes patient data from $>1,500$ accredited facilities and captures $>70 \%$ of newly diagnosed cancer cases in the United States. ${ }^{5}$ The data used in this study were derived from a deidentified NCDB file. The American College of Surgeons and the Commission on Cancer have not verified and are not responsible for the analytic or statistical methodology used or the conclusions drawn from these data by the investigators.

\section{See JNCCN.org for supplemental online content.}


A total of 55,494 patients diagnosed with vulvar cancer between 2004 and 2015 were identified in the NCDB. Patients were excluded from the study if they had unknown or documented negative pelvic lymph nodes. Furthermore, presence or absence of pelvic nodal involvement and the method of pelvic lymph node identification (clinical, radiologic, biopsy, or lymphadenectomy) are cancer-specific factors that were captured within the vulvar cancer database beginning in 2009, with inconsistent documentation before 2009; therefore, patients diagnosed before 2009 were excluded. All patients in this analysis were diagnosed in 2009 through 2015. We confirmed that all patients were staged based on the 8th edition of the AJCC Cancer Staging Manual, in line with the FIGO 2009 staging.

Patients were also excluded if they had known evidence of lung, liver, bone, or brain metastases; previous treatment with RT or chemotherapy was unknown; they received brachytherapy alone; or they had documented RT to a nonpelvic site. Patients diagnosed or treated at a Commission on Cancer-accredited reporting facility were included. The cohort was stratified into those who received surgery and those who did not (Figure 1). Additional stratification included use of RT or chemotherapy. The primary endpoint was OS.

\section{Variable Definitions}

All variables were selected a priori. Sociodemographic variables, including patient age, year of diagnosis (2009-2015), comorbidity score, race/ethnicity, insurance status, median income, distance from facility, facility type, and facility location, were defined according to their respective data fields in the NCDB data dictionary. ${ }^{6}$ Comorbidity was defined based on the Charlson/Deyo comorbidity score. ${ }^{7}$ Race was categorized as non-Hispanic white, black, Hispanic, or other. Insurance status was grouped into government (Medicare/Medicaid/other), private, or uninsured. Income was coded based on census tract estimates and was not patient-specific. Distance from treatment facility to residence was grouped at either $\leq 10$ or $>10$ miles straight-line distance. Facility locations were grouped into Midwest, Northeast, South, and West regions.

Pathologic factors, including tumor size, clinical nodal stage, and grade, were defined according to the data fields. Cancer-specific factors were also evaluated, including extent of primary disease (including depth of invasion when relevant to primary tumor staging). For multivariate analysis (MVA), all T1 lesions were combined and analyzed as 1 value within the T-stage covariate due to small sample size. Tumor size was grouped with a cutoff of $4 \mathrm{~cm}$ to differentiate between larger and smaller $\mathrm{T} 2$ lesions. ${ }^{3}$ Only used as a covariate in surgical patients, number of regional lymph nodes examined and presence of positive regional lymph nodes were grouped as extrapolations of pathologic nodal staging. There is uncertainty

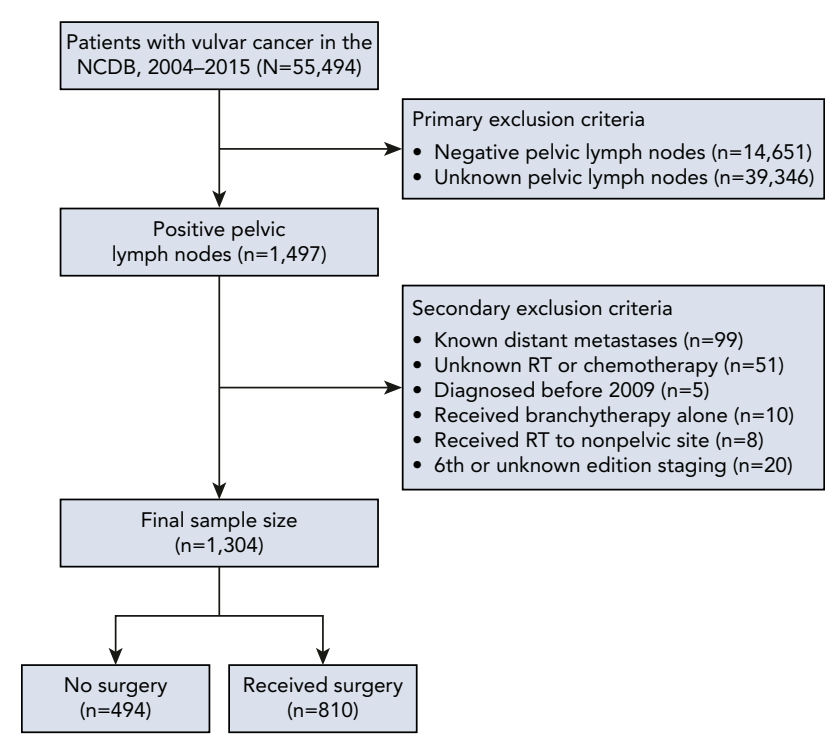

Figure 1. Diagram illustrating exclusion criteria and case selection for the patient cohort.

Abbreviations: NCDB, National Cancer Database; RT, radiation therapy.

within the NCDB coding for vulvar cancer regarding whether pelvic lymph nodes are defined as regional or nonregional, and therefore we omitted distinction between inguinal and pelvic lymph node dissection in the setting of regional lymph node evaluation.

Treatment factors included whether the patient received RT, chemotherapy, or surgery. Extent of surgery was defined as either radical, including total removal plus organ resection, or other, encompassing local excision or extent unknown. Patients documented as undergoing biopsy alone were not defined as undergoing surgery. For MVA, all surgery was combined, turning surgery into a binary covariate similar to receipt of chemotherapy or RT. Concurrent chemotherapy and RT was defined as starting within 2 weeks of each another.

\section{Statistical Analysis}

Descriptive statistics for each of the sociodemographic, pathologic, and treatment variables were summarized based on treatment received. OS was plotted using the Kaplan-Meier method, with differences across groups assessed using the log-rank statistic. Survival time was calculated from date of diagnosis until date of death or last contact if alive. Patients alive at last contact were censored at last contact date. Conditional landmark analysis was performed, evaluating only patients who survived at least 60 days from initial diagnosis to allow completion of any planned adjuvant treatment.

Survival analyses were performed using the log-rank test for univariate analysis and Cox proportional hazards regression for MVA. Initial variable selection included all variables discussed previously. Patients with unknown 
variables had "unknown" entered as a dummy value to allow analysis without further loss of sample size. The final parsimonious multivariate Cox model was formed by using multivariate hierarchical backward selection of variables at a significance level of $P<.10$. Proportional hazards assumption was assessed for all variables in the final MVA and was not violated. ${ }^{8}$ Additional MVA Cox models were developed for subset analyses in patients undergoing or not undergoing surgery, using the same method as described earlier. A multivariate logistic regression was performed to assess factors predictive of undergoing surgery and RT using multivariate hierarchical backward selection of variables at a significance level of $P<.10$. An alpha threshold of 0.05 was chosen for statistical significance. Statistical analyses were performed using SPSS Statistics, version 24.0 (IBM Corp).

\section{Results}

\section{Patient Characteristics}

A total of 1,304 patients who met inclusion criteria were identified in the NCDB (Figure 1). Median follow-up was 21 months (38 months for survivors). Table 1 summarizes the sociodemographic, pathologic, and treatment characteristics of the study cohort, stratifying and comparing baseline differences between the surgery and no surgery groups. Significant baseline differences were seen, primarily smaller tumor size and lower clinical nodal stage in patients undergoing surgery.

Patients undergoing surgery were more likely to be treated before 2013, have comorbidities, identify as white, have private insurance, live $>10$ miles from a treatment facility, and have lower tumor stage, smaller tumors, and a lower clinical nodal stage; they were also less likely to receive chemotherapy or RT. For all patients, 3 -year and 5-year OS rates were $46.2 \%$ and $37.1 \%$, respectively.

\section{Comparison of Surgery Use and Associated Outcomes}

Of the 1,304 patients included, 810 (62.1\%) underwent surgical resection. Factors reliant on surgery, such as evaluation of regional lymph nodes and positive nodal count, were not included. Factors that were significant on multivariate logistic regression for higher likelihood of surgery included younger age, treatment before 2013, white race, lower tumor stage, and clinically node-negative disease (Table 2).

Median follow-up in patients who did not undergo surgery was 15 months (34.5 months for survivors) compared with 24 months (39 months for survivors) in those who did undergo surgery. Survival outcomes favored patients undergoing surgery on univariate analysis, with 3 -year OS of $53.2 \%$ versus $32.4 \%$ in patients not undergoing surgery $(P<.001)$ (Figure 2). MVA revealed that receipt of surgery was associated with prolonged OS (hazard ratio [HR], 0.58; 95\% CI, 0.48-0.72; $P<.001$ ) (Table 3). Receipt of RT was also associated with prolonged OS (HR, 0.75; 95\% CI, 0.62-0.92; $P=.004)$. Other factors associated with prolonged OS included younger age, lack of comorbidities, uninsured, treatment in an academic/research facility, smaller tumor size $(<4.0 \mathrm{~cm})$, and being clinically nodenegative (all $P<.05$ ).

\section{Use of RT and Outcomes in Patients}

\section{Undergoing Surgery}

When evaluating only the 810 patients who underwent surgical resection, we observed that $572(70.6 \%)$ also underwent RT (Table 1). In MVA, factors significant for increased likelihood of RT use in these patients included more recent year of diagnosis, distance of $\leq 10$ miles from facility, treatment at a community facility, having 2 or 3 positive lymph nodes at time of surgery, and receipt of chemotherapy (all $P<.05$ ) (supplemental eTable 1, available with this article at JNCCN.org).

Survival analyses of patients undergoing surgery showed that addition of RT was associated with prolonged OS (3-year OS, $55.4 \%$ vs $48.0 \%$ without RT; $P=.033$ ) (Figure $3 \mathrm{~A}$ ). The association of RT with prolonged OS was maintained in MVA (HR, 0.78; $P=.048$ ) (supplemental eTable 2). Other factors associated with prolonged OS included younger age, lack of comorbidities, smaller tumor size, and having no positive lymph nodes (all $P<.05$ ). Use of chemotherapy was not associated with survival in this patient population.

\section{Outcomes in Patients Not Undergoing Surgery}

Of 494 patients who did not undergo surgery, 389 (78.7\%) received RT. Most patients were treated with both chemotherapy + RT ( $\mathrm{n}=299 ; 60.5 \%)$ (Table 1$)$. RT was associated with prolonged survival in univariate analysis (3-year OS, $35.5 \%$ vs $17.1 \%$ in patients not receiving RT; $P<.001$ ) (Figure 3B). Use of RT and chemotherapy was associated with prolonged OS in MVA (HR, $0.66 ; P=.019$ ) (supplemental eTable 3). Other factors associated with prolonged OS included younger age, nongovernment insurance, and tumors $\leq 4 \mathrm{~cm}$. Receipt of chemotherapy did not significantly improve OS in this patient population.

\section{Timing of Surgery, RT, and Chemotherapy}

A total of 572 patients received both surgery and RT, 550 (96.2\%) of whom had evaluable time point data for both variables. Among patients who received surgery and RT, $90 \%$ received RT after surgical resection. A total of 643 patients received both chemotherapy and RT, 621 (96.6\%) of whom were evaluable. Concurrent treatment was the most common method of combining these modalities, used in $85 \%$ of the studied patients. 


\section{Table 1. Baseline Characteristics of Study Cohort}

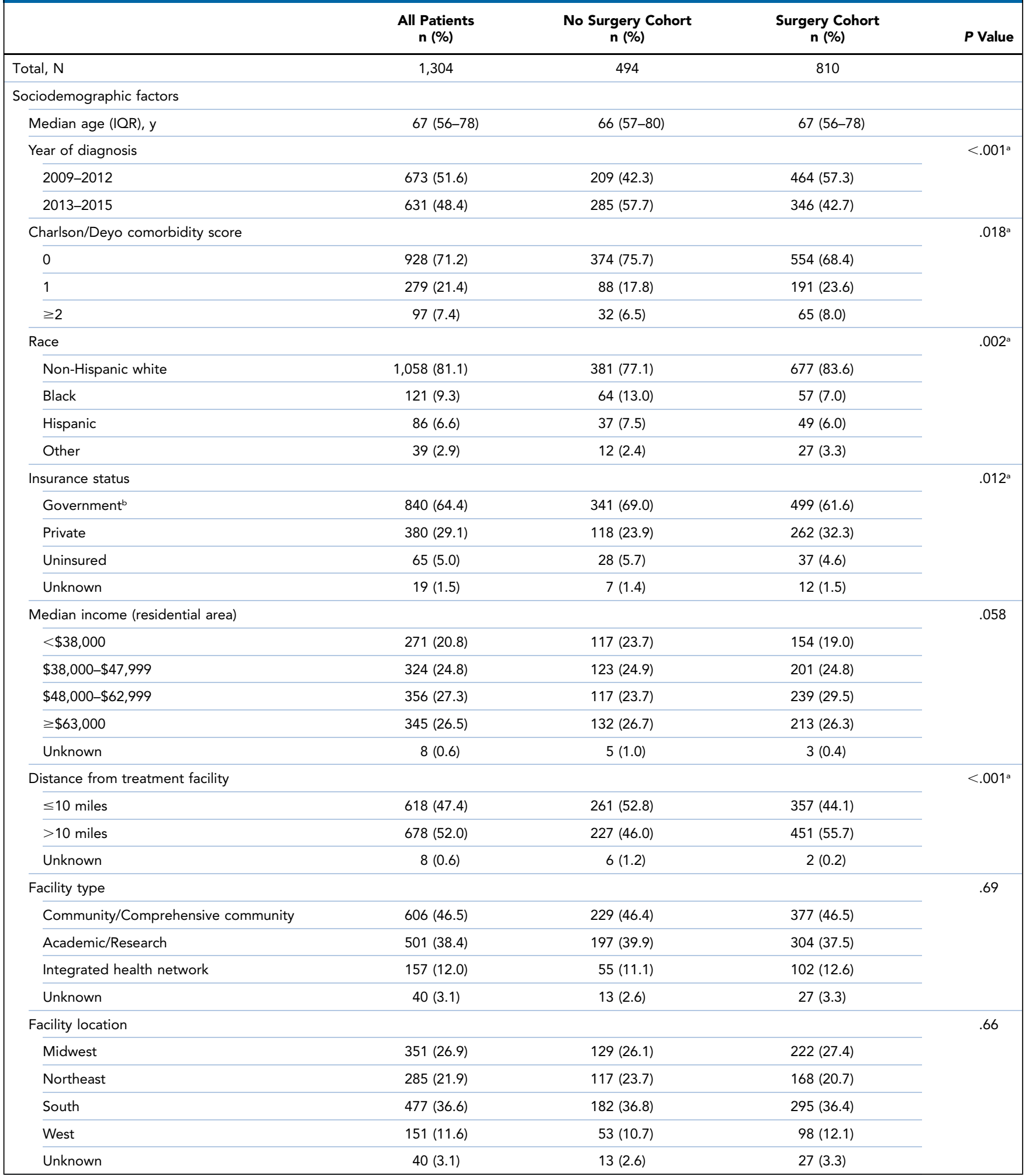

Abbreviations: $\mathrm{DOI}$, depth of invasion; IQR, interquartile range; $\mathrm{RT}$, radiation therapy.

bIncludes Medicare, Medicaid, and other. 
Table 1. Baseline Characteristics of Study Cohort (cont.)

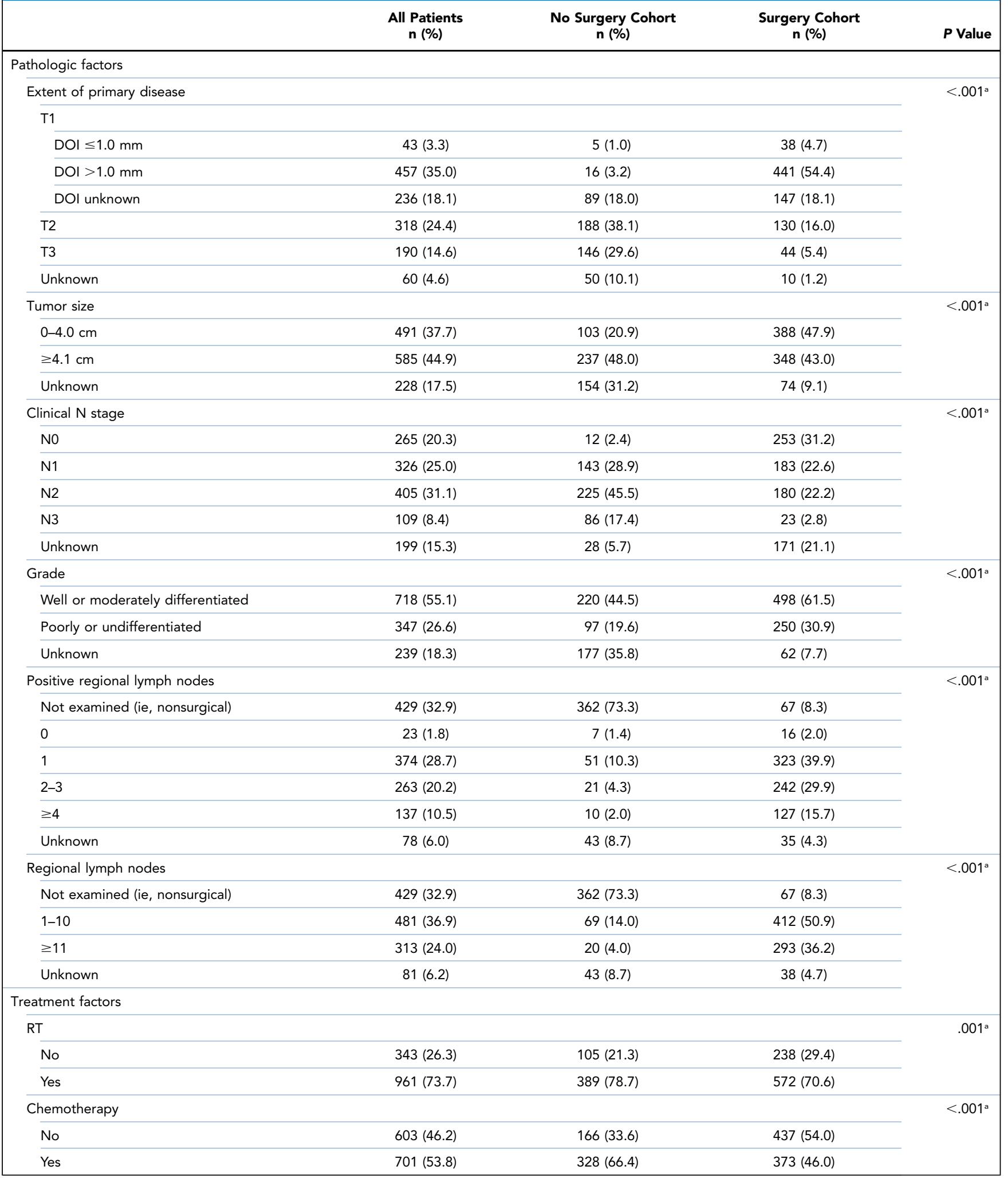

Abbreviations: DOI, depth of invasion; IQR, interquartile range; $\mathrm{RT}$, radiation therapy

(continued on next page)

bIncludes Medicare, Medicaid, and other. 


\begin{tabular}{|c|c|c|c|c|}
\hline Surgery & & & & $<.001^{a}$ \\
\hline None & 494 (37.9) & $494(100)$ & $0(0)$ & \\
\hline Other surgery & $375(28.8)$ & $0(0)$ & $375(46.3)$ & \\
\hline Treatment combinations & & & & $<.001^{\text {a }}$ \\
\hline Observation & $76(5.8)$ & $76(15.4)$ & $0(0)$ & \\
\hline RT alone & $90(6.9)$ & $90(18.2)$ & $0(0)$ & \\
\hline Chemotherapy alone & $29(2.2)$ & $29(5.9)$ & $0(0)$ & \\
\hline Chemotherapy + RT & 299 (22.9) & $299(60.5)$ & $0(0)$ & \\
\hline Surgery + chemotherapy + RT & $344(26.3)$ & $0(0)$ & $344(42.5)$ & \\
\hline
\end{tabular}

Abbreviations: DOI, depth of invasion; IQR, interquartile range; $R T$, radiation therapy.

aStatistically significant.

bIncludes Medicare, Medicaid, and other.

\section{Discussion}

This study shows that in patients with vulvar cancer metastatic to pelvic lymph nodes, consideration should be given to definitive management using $\geq 1$ locoregional treatment. Patients who underwent surgery had significantly different baseline characteristics from those who did not, limiting the possible conclusions about benefit of surgery, even with standard methods of limiting confounding, such as propensity score adjustment or propensity score matching. If patients are surgically resectable, consideration could be given to surgical therapy. Patients who underwent surgical resection seemed to benefit from adjuvant RT; however, adjuvant chemotherapy appeared to have no effect. Patients who did not undergo surgical resection seemed to benefit most from RT. The role of chemotherapy in either surgical or nonsurgical patients was not well elucidated in this study. Although no statistically significant improvement in OS was seen with addition of chemotherapy, this may be due more to a heterogeneous patient population and limitations of sample size than to a true lack of benefit of chemotherapy.

Previous research determining the role of definitive treatment in patients with vulvar cancer with limited metastases to pelvic lymph nodes is limited to a small single-institution series of 20 patients treated with combinations of surgery, RT, and chemoradiation, showing 5 -year OS of $43 \% .{ }^{4}$ Our study shows similar outcomes when evaluating all patients. The question posed by Thaker et $\mathrm{al}^{4}$ regarding reconsideration of the FIGO staging system is a relevant one, echoed in our study.
Multiple studies in vulvar cancer show significantly worse outcomes in patients with regionally node-positive disease, with 2- to 5-year survival outcomes ranging between $30 \%$ and $60 \% .^{9-12}$ These previously published rates of OS are similar to the 3- and 5-year OS rates of $46.2 \%$ and $37.1 \%$, respectively, seen in our study. Patients who underwent both surgery and RT also had a 3-year OS rate as high as 55\%. Additionally, patients with positive pelvic lymph nodes seemed to have better survival than the 2-year OS of approximately $10 \%$ reported in those with distant metastases. ${ }^{13}$

Current management of vulvar cancer in either the definitive, neoadjuvant, or postoperative setting includes purposeful RT of lower pelvic lymph nodes, including obturator, external iliac, and internal iliac, when nodal volumes are being treated. ${ }^{14}$ The superior aspect of the pelvic RT field, however, is slightly more nebulous and dependent on clinical scenario. In GOG 37, RT went as high as the midpelvis, ${ }^{12}$ and in GOG 101 to the inferior sacroiliac joint. ${ }^{15}$ The ongoing GROINSS-V II/GOG 270 study of postoperative vulvar cancer (ClinicalTrials.gov identifier: NCT01500512) mandates coverage of the lower half of the external iliac lymph nodes when lymph node RT is performed. Given that coverage of pelvic lymph nodes is routine in the prophylactic setting, treatment of involved pelvic lymph nodes would be consistent with the treatment approach of other pelvic malignancies, such as anal and cervical cancer.

Increased knowledge of cancer biology, together with improvements in both systemic and locoregional therapy, has redefined patients previously considered to have metastatic disease in multiple disease sites. 
Table 2. Multivariate Analysis of Use of Surgery by Baseline Characteristics

Odds Ratio $(98 \% \mathrm{Cl})$

$P$ Value

Total, $\mathrm{N}$
Sociodemographic factors

\begin{tabular}{lll}
\hline Median age (IQR), y & $0.99(0.98-1.00)$ & .043
\end{tabular}

Year of diagnosis

2009-2012 Ref

2013-2015 $0.45(0.32-0.61)<.001^{\mathrm{b}}$

Race

\begin{tabular}{lcc}
\hline Non-Hispanic white & Ref & \\
\hline Black & $0.49(0.29-0.84)$ & .009 \\
\hline Hispanic & $0.75(0.39-1.44)$ & .38 \\
\hline Other & $1.38(0.52-3.62)$ & .52
\end{tabular}

Distance from treatment facility

\begin{tabular}{lcc}
$\leq 10$ miles & Ref & \\
\hline$>10$ miles & $1.35(0.98-1.87)$ & .071 \\
Unknown & $0.18(0.19-1.64)$ & .13
\end{tabular}

Pathologic factors

Extent of primary disease

\begin{tabular}{lcc}
\hline T1 & Ref & \\
\hline T2 & $0.210(0.15-0.30)$ & $<.001^{\text {b }}$ \\
\hline T3 & $0.083(0.05-0.13)$ & $<.001^{\mathrm{b}}$ \\
\hline Unknown & $0.070(0.03-0.16)$ & $<.001^{\mathrm{b}}$
\end{tabular}

Tumor size

\begin{tabular}{lcc}
\hline $0-4.0 \mathrm{~cm}$ & Ref & \\
\hline$\geq 4.1 \mathrm{~cm}$ & $0.75(0.52-1.08)$ & .12 \\
\hline Unknown & $0.29(0.18-0.46)$ & $<.001^{\mathrm{b}}$
\end{tabular}

Clinical N stage

\begin{tabular}{lcc}
\hline N0 & Ref & \\
\hline $\mathrm{N} 1$ & $0.094(0.05-0.19)$ & $<.001^{\mathrm{b}}$ \\
\hline $\mathrm{N} 2$ & $0.073(0.04-0.14)$ & $<.001^{\mathrm{b}}$ \\
\hline $\mathrm{N} 3$ & $0.030(0.01-0.07)$ & $<.001^{\mathrm{b}}$ \\
\hline Unknown & $0.480(0.22-1.07)$ & .071 \\
\hline Grade & Ref & \\
\hline Well or moderately differentiated & $1.30(0.89-1.89)$ & .18 \\
\hline Poorly or undifferentiated & $0.28(0.18-0.42)$ & $<.001^{\mathrm{b}}$ \\
\hline Unknown & &
\end{tabular}

Abbreviation: IQR, interquartile range.

aOdds ratio $<1$ : less likely to undergo surgery; odds ratio $>1$ : more likely to undergo surgery.

bStatistically significant.

Cervical cancer with para-aortic lymph node involvement used to be considered M1 disease, ${ }^{16}$ but multiple studies have shown good oncologic outcomes with definitive local treatment, ${ }^{17-19}$ resulting in an eventual shift of paraaortic lymph node involvement to be considered regional
Table 3. Multivariable Cox Proportional Hazards Model for Overall Survival in All Patients

Hazard Ratio
$(95 \% \mathrm{Cl})$$\quad P$ Value

Sociodemographic factors

Age

$1.03(1.02-1.04)<.001^{\text {a }}$

Charlson/Deyo comorbidity score

0

1

Ref

1

$1.26(1.03-1.54) \quad .025^{\mathrm{a}}$

$\geq 2$

$1.45(1.06-1.99) \quad .020^{a}$

Insurance status

Government ${ }^{\mathrm{b}}$

Ref

Private

$0.82(0.66-1.02) \quad .079$

Uninsured

$0.52(0.30-0.88) \quad .015^{\mathrm{a}}$

Unknown

$0.40(0.15-1.07) \quad .068$

Median income (residential area)

$<\$ 38,000$

Ref

$\$ 38,000-\$ 47,999$

$0.83(0.65-1.06) \quad .140$

$\$ 48,000-\$ 62,999$

$0.77(0.60-0.98) \quad .033^{a}$

$\geq \$ 63,000$

$0.91(0.71-1.15) \quad .420$

Unknown

$2.26(1.03-4.95) \quad .042$

Facility type

Community/Comprehensive community

Ref

Academic/Research

$0.78(0.65-0.93) \quad .006^{a}$

Integrated health network

$0.87(0.66-1.14) \quad .300$

Unknown

$1.70(0.91-3.17) \quad .094$

Pathologic factors

Tumor size

$0-4.0 \mathrm{~cm} \quad$ Ref

$\geq 4.1 \mathrm{~cm}$

$1.62(1.33-1.96)<.001^{a}$

Unknown

1.15 (0.89-1.49) .280

Clinical $\mathrm{N}$ stage

NO

Ref

N1

1.37 (1.04-1.79) $\quad .024^{\mathrm{a}}$

N2

$1.37(1.04-1.82) \quad .026^{a}$

N3

$1.66(1.13-2.43) \quad .010^{a}$

Unknown

1.25 (0.93-1.69) . .140

Treatment factors

Radiation therapy

\begin{tabular}{ccc}
\hline No & Ref & \\
\hline Yes & $0.75(0.62-0.92)$ & $.004^{\text {a }}$
\end{tabular}

Surgery

No

Ref

Yes

$0.58(0.48-0.72)<.001^{a}$

aStatistically significant.

bIncludes Medicare, Medicaid, and other. 


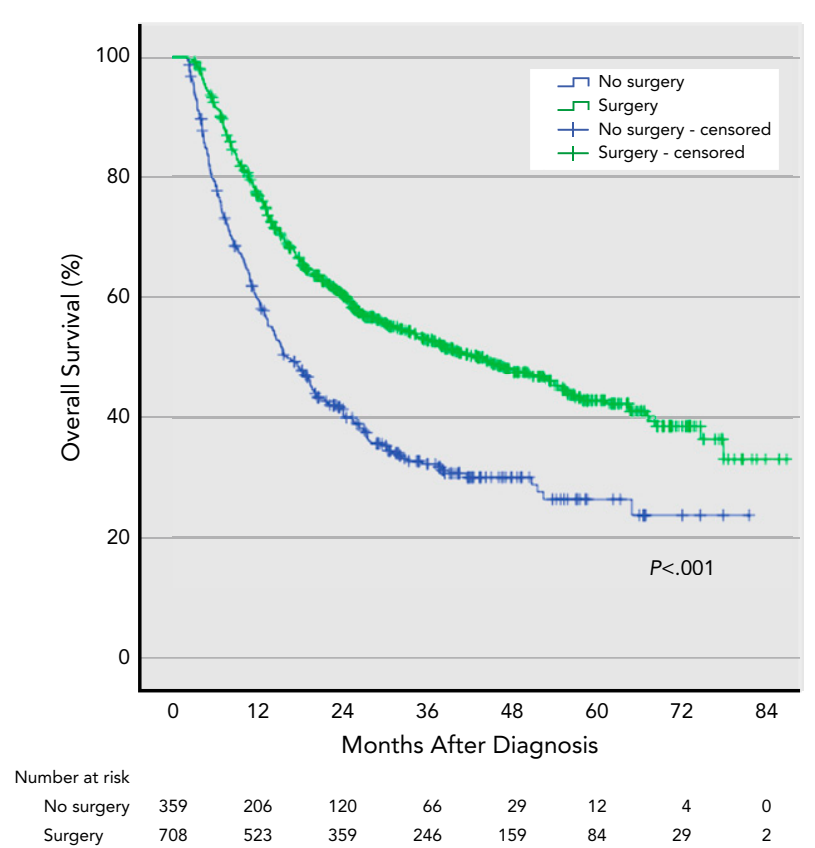

Figure 2. Kaplan-Meier overall survival curve, stratified by receipt of surgery, for patients with $\geq 60$ days of follow up.

nodal disease. ${ }^{2}$ Similar findings have been shown in nodepositive ${ }^{20-22}$ and distant metastatic prostate cancer. ${ }^{23}$

The role for definitive RT, generally with chemotherapy, in the management of unresectable vulvar cancer is well documented from multiple prospective studies, ${ }^{15,24}$ with contemporary series showing feasibility of using advanced techniques with good oncologic outcomes in well-selected patients. ${ }^{25-27}$ Standard limitations of retrospective analysis and database studies apply. We attempted to mitigate as much bias as possible by adjusting for measured confounders through MVA. However, given the NCDB limitations and potential for ascertainment bias, we believed that there was too extreme a difference in baseline characteristics between patients undergoing surgery and those not undergoing surgery. Even with potentially conducting propensity score matching, we were not confident that we would be able to control sufficiently for other confounding variables that may not be as well-captured within the NCDB, such as more sensitive markers of morbidity, surgeon's assessment of resectability, and nature of lymph node disease, among others. Given that pelvic lymph node status did not seem to be regularly coded until 2009, the sample size was somewhat limited, given the clinical rarity of this disease stage and that $>70 \%$ of patients with vulvar cancer captured in the NCDB have an unknown pelvic lymph node status. Missing data were an issue for certain examined variables, such as primary tumor size and grade, which were both unknown in approximately $30 \%$ of patients not undergoing surgery. The NCDB does not capture which lymph node basins are covered by RT, nor does it reliably capture whether the primary tumor was treated. Given these significant concerns, to suggest a causative relationship or comparative effectiveness of up-front surgery versus up-front RT is beyond the ability of the database and the scope of this paper. However, some form of definitive locoregional treatment, either RT or surgery (with consideration of adjuvant RT), rather than treating with palliative chemotherapy alone, seems to benefit patients in this clinical scenario.

\section{A}
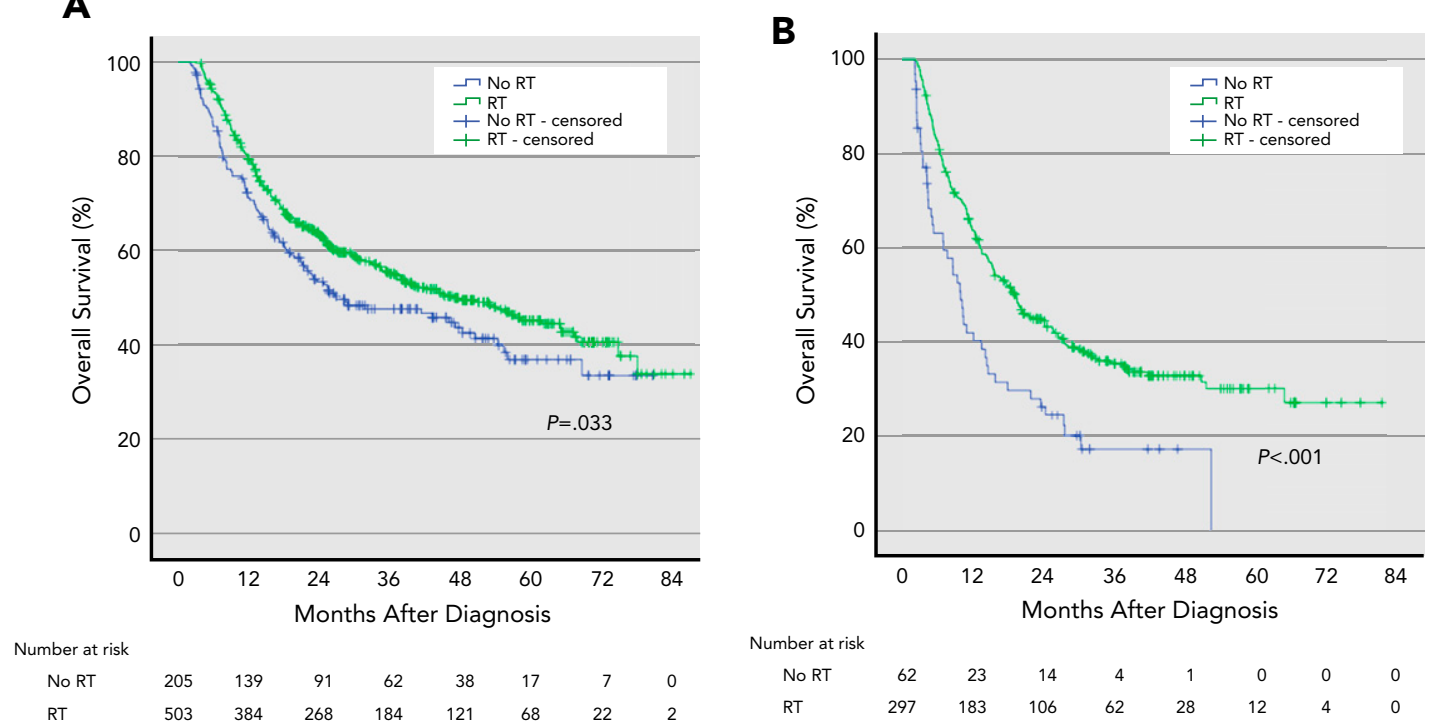

Figure 3. Kaplan-Meier overall survival curve, stratified by receipt of RT, in patients (A) undergoing surgery and (B) not undergoing surgery. Abbreviation: RT, radiation therapy. 
However, because this is the largest series to evaluate this relevant endpoint and given the lethality of nodepositive vulvar cancer, we believe that these findings are interesting enough to consider future prospective clinical trials in this clinical scenario. In the absence of randomized data, we believe that the 9th edition of the AJCC Cancer Staging Manual should consider whether pelvic lymph node metastases remain classified as a metastatic disease site, given that survival numbers among these patients are much closer to those of patients with regionally nodepositive disease rather than known metastatic disease.

\section{Conclusions}

Patients with vulvar cancer metastatic to pelvic lymph nodes should be strongly considered for aggressive, definitive treatment similar to those with inguinal lymph node-positive disease. Patients with surgically resectable disease should be considered for surgery and adjuvant RT, without strong evidence to support use of concurrent chemotherapy. Patients with either technically or medically unresectable disease should be treated with definitive RT, with consideration of concurrent chemotherapy.

Submitted September 7, 2018; accepted for publication February 21, 2019.

Author contributions: Study concept: Shinde, Li, Amini, Glaser. Data curation: Shinde, Li, Glaser. Methodology: Shinde, Glaser. Drafting of manuscript: Shinde, Glaser. Manuscript review and editing: All authors.

Disclosures: The authors have disclosed that they have not received any financial considerations from any person or organization to support the preparation, analysis, results, or discussion of this article.

Correspondence: Scott Glaser, MD, Department of Radiation Oncology, City of Hope National Cancer Center, 1500 East Duarte Road, Duarte, CA 91010. Email: sglaser@coh.org

\section{References}

1. Siegel RL, Miller KD, Jemal A. Cancer statistics, 2018. CA Cancer J Clin 2018;68:7-30

2. Amin MB, Edge SB, Greene F, et al, eds. AJCC Cancer Staging Manual, 8th ed. New York, NY: Springer International Publishing; 2017.

3. Koh WJ, Greer BE, Abu-Rustum NR, et al. NCCN Clinical Practice Guidelines in Oncology: Vulvar Cancer. Version 1.2017. Accessed February 1, 2018. To view the most recent version, visit NCCN.org

4. Thaker NG, Klopp AH, Jhingran A, et al. Survival outcomes for patients with stage IVB vulvar cancer with grossly positive pelvic lymph nodes: time to reconsider the FIGO staging system? Gynecol Oncol 2015;136: 269-273.

5. Bilimoria KY, Stewart AK, Winchester DP, et al. The National Cancer Data Base: a powerful initiative to improve cancer care in the United States. Ann Surg Oncol 2008;15:683-690.

6. American College of Surgeons. National Cancer Database: participant user file data dictionary. Available at: http://ncdbpuf.facs.org. Accessed March 26, 2018

7. Quan H, Li B, Couris CM, et al. Updating and validating the Charlson comorbidity index and score for risk adjustment in hospital discharge abstracts using data from 6 countries. Am J Epidemiol 2011;173:676-682.

8. Bellera CA, MacGrogan G, Debled M, et al. Variables with time-varying effects and the Cox model: some statistical concepts illustrated with a prognostic factor study in breast cancer. BMC Med Res Methodol 2010; 10:20.

9. Nooij LS, Ongkiehong PJ, van Zwet EW, et al. Groin surgery and risk of recurrence in lymph node positive patients with vulvar squamous cell carcinoma. Gynecol Oncol 2015;139:458-464.

10. Mahner S, Jueckstock J, Hilpert F, et al. Adjuvant therapy in lymph node-positive vulvar cancer: the AGO-CaRE-1 study. J Natl Cancer Inst 2015;107:dju426.

11. Woelber L, Eulenburg C, Choschzick M, et al. Prognostic role of lymph node metastases in vulvar cancer and implications for adjuvant treatment. Int J Gynecol Cancer 2012;22:503-508.

12. Homesley HD, Bundy BN, Sedlis A, et al. Radiation therapy versus pelvic node resection for carcinoma of the vulva with positive groin nodes. Obstet Gynecol 1986;68:733-740.

13. Prieske K, Haeringer N, Grimm D, et al. Patterns of distant metastases in vulvar cancer. Gynecol Oncol 2016;142:427-434.

14. Gaffney DK, King B, Viswanathan AN, et al. Consensus recommendations for radiation therapy contouring and treatment of vulvar carcinoma. Int J Radiat Oncol Biol Phys 2016;95:1191-1200

15. Moore DH, Ali S, Koh WJ, et al. A phase II trial of radiation therapy and weekly cisplatin chemotherapy for the treatment of locally-advanced

squamous cell carcinoma of the vulva: a Gynecologic Oncology Group study. Gynecol Oncol 2012;124:529-533.

16. Edge SB, Byrd DR, Compton CC, et al, eds. AJCC Cancer Staging Manual 7th ed. New York, NY: Springer; 2010.

17. Beriwal S, Gan GN, Heron DE, et al. Early clinical outcome with concurren chemotherapy and extended-field, intensity-modulated radiotherapy for cervical cancer. Int J Radiat Oncol Biol Phys 2007;68:166-171.

18. Vargo JA, Kim H, Choi S, et al. Extended field intensity modulated radiation therapy with concomitant boost for lymph node-positive cervical cancer: analysis of regional control and recurrence patterns in the positron emission tomography/computed tomography era. Int J Radiat Oncol Biol Phys 2014;90:1091-1098.

19. Lindegaard JC, Assenholt M, Ramlov A, et al. Early clinical outcome of coverage probability based treatment planning for simultaneous integrated boost of nodes in locally advanced cervical cancer. Acta Oncol 2017;56:1479-1486.

20. Abdollah F, Karnes RJ, Suardi N, et al. Impact of adjuvant radiotherapy on survival of patients with node-positive prostate cancer. J Clin Oncol 2014;32:3939-3947.

21. Gupta M, Patel HD, Schwen ZR, et al. Adjuvant radiation with androgendeprivation therapy for men with lymph node metastases after radical prostatectomy: identifying men who benefit. BJU Int 2019;123:252-260.

22. Bryant AK, Kader AK, McKay RR, et al. Definitive radiation therapy and survival in clinically node-positive prostate cancer. Int J Radiat Oncol Biol Phys 2018;101:1188-1193.

23. Rusthoven CG, Jones BL, Flaig TW, et al. Improved survival with prostate radiation in addition to androgen deprivation therapy for men with newly diagnosed metastatic prostate cancer. J Clin Oncol 2016;34:2835-2842.

24. Moore DH, Thomas GM, Montana GS, et al. Preoperative chemoradiation for advanced vulvar cancer: a phase II study of the Gynecologic Oncology Group. Int J Radiat Oncol Biol Phys 1998;42:79-85.

25. Beriwal S, Shukla G, Shinde A, et al. Preoperative intensity modulated radiation therapy and chemotherapy for locally advanced vulvar carcinoma: analysis of pattern of relapse. Int J Radiat Oncol Biol Phys 2013;85:1269-1274.

26. Stecklein SR, Frumovitz M, Klopp AH, et al. Effectiveness of definitive radiotherapy for squamous cell carcinoma of the vulva with gross inguinal lymphadenopathy. Gynecol Oncol 2018;148:474-479.

27. Rao YJ, Chundury A, Schwarz JK, et al. Intensity modulated radiation therapy for squamous cell carcinoma of the vulva: treatment technique and outcomes. Adv Radiat Oncol 2017;2:148-158.

See JNCCN.org for supplemental online content. 
Supplemental online content for:

\section{Role of Locoregional Treatment in Vulvar Cancer With Pelvic Lymph Node Metastases: Time to Reconsider FIGO Staging?}

Ashwin Shinde, MD; Richard Li, MD; Arya Amini, MD; Yi-Jen Chen, MD, PhD; Mihaela Cristea, MD; Wenge Wang, MD, PhD; Mark Wakabyashi, MD; Ernest Han, MD, PhD; Catheryn Yashar, MD; Kevin Albuquerque, MD; Sushil Beriwal, MD; and Scott Glaser, MD

J Natl Compr Canc Netw 2019;17(8):922-930

eTable 1: Multivariate Analysis of Use of RT in Patients Who Underwent Surgery eTable 2: Multivariable Cox Proportional Hazards Model for Overall Survival in Patients Who Underwent Surgery eTable 3: Multivariable Cox Proportional Hazard Models for Overall Survival in Patients Who Did Not Undergo Surgery 


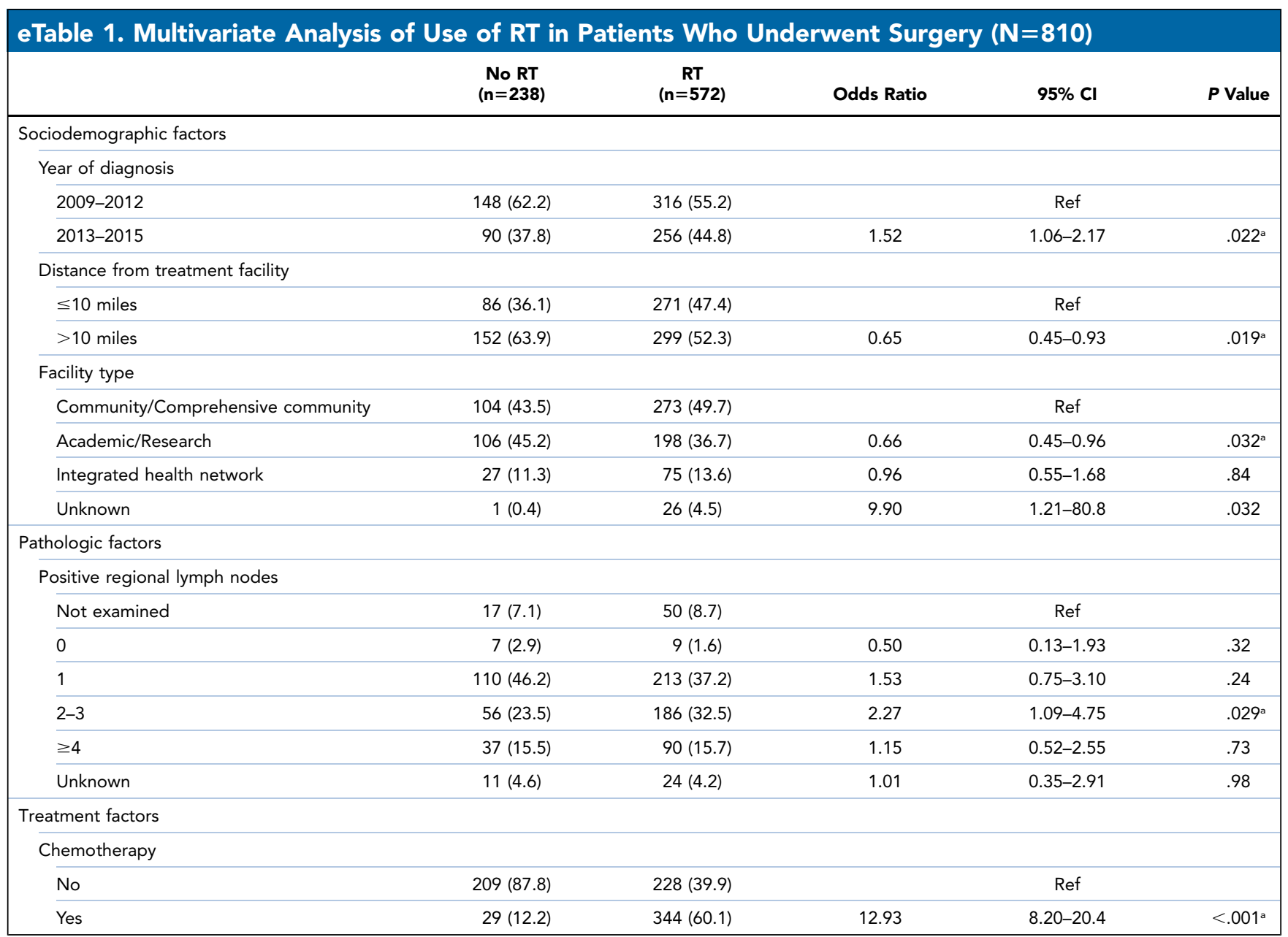

Abbreviation: RT, radiation therapy.

aStatistically significant. 
eTable 2. Multivariable Cox Proportional Hazards Model for Overall Survival in Patients

Who Underwent Surgery

\begin{tabular}{|c|c|c|}
\hline & $\begin{array}{c}\text { Hazard Ratio } \\
(95 \% \mathrm{Cl})\end{array}$ & $P$ Value \\
\hline \multicolumn{3}{|l|}{ Sociodemographic factors } \\
\hline Age & $1.04(1.03-1.05)$ & $<.001^{\text {a }}$ \\
\hline \multicolumn{3}{|l|}{ Charlson/Deyo comorbidity score } \\
\hline 0 & Ref & \\
\hline 1 & $1.41(1.10-1.81)$ & $.007^{\mathrm{a}}$ \\
\hline$\geq 2$ & $1.82(1.26-2.62)$ & $.001^{\mathrm{a}}$ \\
\hline \multicolumn{3}{|l|}{ Facility type } \\
\hline Community/Comprehensive community & Ref & \\
\hline Academic/Research & $0.79(0.62-1.00)$ & .050 \\
\hline Integrated health network & $0.78(0.54-1.12)$ & .18 \\
\hline \multicolumn{3}{|l|}{ Pathologic factors } \\
\hline \multicolumn{3}{|l|}{ Tumor size } \\
\hline $0-4.0 \mathrm{~cm}$ & Ref & \\
\hline$\geq 4.1 \mathrm{~cm}$ & $1.60(1.27-2.02)$ & $<.001^{\text {a }}$ \\
\hline Unknown & $0.88(0.57-1.34)$ & .54 \\
\hline \multicolumn{3}{|l|}{ Positive regional lymph nodes } \\
\hline Not examined & Ref & \\
\hline 0 & $0.24(0.05-1.26)$ & $.092^{\mathrm{a}}$ \\
\hline 1 & $0.94(0.37-2.36)$ & .891 \\
\hline $2-3$ & $1.64(0.66-4.11)$ & .29 \\
\hline$\geq 4$ & $2.84(1.11-7.27)$ & $.030^{\mathrm{a}}$ \\
\hline Unknown & $1.43(0.77-2.67)$ & .26 \\
\hline \multicolumn{3}{|l|}{ Treatment factors } \\
\hline \multicolumn{3}{|l|}{ Radiation therapy } \\
\hline No & Ref & \\
\hline Yes & $0.78(0.61-0.99)$ & $.048^{a}$ \\
\hline
\end{tabular}

aStatistically significant.

\begin{tabular}{|c|c|c|}
\hline \multicolumn{3}{|c|}{$\begin{array}{l}\text { eTable 3. Multivariable Cox Proportional Hazard } \\
\text { Models for Overall Survival in Patients } \\
\text { Who Did Not Undergo Surgery }\end{array}$} \\
\hline & $\begin{array}{c}\text { Hazard Ratio } \\
\text { (95\% Cl) }\end{array}$ & $P$ Value \\
\hline \multicolumn{3}{|c|}{ Sociodemographic factors } \\
\hline Age & $1.03(1.01-1.04)$ & $<.001^{a}$ \\
\hline \multicolumn{3}{|l|}{ Insurance status } \\
\hline Government & Ref & \\
\hline Private & $0.52(0.36-0.76)$ & $.001^{a}$ \\
\hline Uninsured & $0.48(0.24-0.97)$ & $.041^{\mathrm{a}}$ \\
\hline Unknown & $0.30(0.07-1.21)$ & .089 \\
\hline \multicolumn{3}{|c|}{ Median income (residential area) } \\
\hline$<\$ 38,000$ & Ref & \\
\hline$\$ 38,000-\$ 47,999$ & $0.60(0.41-0.90)$ & $.013^{\mathrm{a}}$ \\
\hline$\$ 48,000-\$ 62,999$ & $0.74(0.50-1.08)$ & .12 \\
\hline$\geq \$ 63,000$ & $0.70(0.48-1.01)$ & .057 \\
\hline Unknown & $1.65(0.64-4.28)$ & .30 \\
\hline \multicolumn{3}{|l|}{ Pathologic factors } \\
\hline \multicolumn{3}{|l|}{ Tumor size } \\
\hline $0-4.0 \mathrm{~cm}$ & Ref & \\
\hline$\geq 4.1 \mathrm{~cm}$ & $1.50(1.04-2.15)$ & $.029^{a}$ \\
\hline Unknown & $1.37(0.94-2.00)$ & .099 \\
\hline \multicolumn{3}{|l|}{ Treatment factors } \\
\hline \multicolumn{3}{|l|}{ Radiation therapy } \\
\hline No & Ref & \\
\hline Yes & $0.66(0.47-0.94)$ & $.019^{a}$ \\
\hline
\end{tabular}

aStatistically significant. 